\title{
Improving fold resistance prediction of HIV- 1 against protease and reverse transcriptase inhibitors using artificial neural networks
}

\author{
Olivier Sheik Amamuddy ${ }^{1}$, Nigel T. Bishop ${ }^{2}$ and Özlem Tastan Bishop ${ }^{1 *}$ (D)
}

\begin{abstract}
Background: Drug resistance in HIV treatment is still a worldwide problem. Predicting resistance to antiretrovirals (ARVs) before starting any treatment is important. Prediction accuracy is essential, as low-accuracy predictions increase the risk of prescribing sub-optimal drug regimens leading to patients developing resistance sooner. Artificial Neural Networks (ANNs) are a powerful tool that would be able to assist in drug resistance prediction. In this study, we constrained the dataset to subtype B, sacrificing generalizability for a higher predictive performance, and demonstrated that the predictive quality of the ANN regression models have definite improvement for most ARVs.

Results: Trained regression ANNs were optimized for eight protease inhibitors, six nucleoside reverse transcriptase (RT) inhibitors and four non-nucleoside RT inhibitors by experimenting combinations of rare variant filtering (none versus 1 residue occurrence) and ANN topologies (1-3 hidden layers with 2, 4, 6, 8 and 10 nodes per layer). Single hidden layers (5-20 nodes) were used for training where overfitting was detected. 5-fold cross-validation produced mean $R^{2}$ values over 0.95 and standard deviations lower than 0.04 for all but two antiretrovirals.

Conclusions: Overall, higher accuracies and lower variances (compared to results published in 2016) were obtained by experimenting with various preprocessing methods, while focusing on the most prevalent subtype in the raw dataset (subtype B).We thus highlight the need to develop and make available subtype-specific datasets for developing higher accuracy in drug-resistance prediction methods.
\end{abstract}

Keywords: Artificial neural network, Drug resistance prediction, Subtype-specific training, HIV-1 subtype B, HIV reverse transcriptase, HIV protease

\section{Background}

Living with HIV has come a long way from being a deadly disease to become a manageable chronic infection [1] mainly due to the development and use of antiretrovirals (ARVs). However, resistance to ARVs still prevails for multiple reasons including non-adherence to treatment, use of sub-optimal regimens and delayed initiation of therapy $[2,3]$. Thus predicting resistance to ARVs before and during any treatment is important, and therefore genotypic testing for prediction finds wide

\footnotetext{
* Correspondence: O.TastanBishop@ru.ac.za

${ }^{1}$ Research Unit in Bioinformatics (RUBi), Department of Biochemistry and

Microbiology, Rhodes University, Grahamstown 6140, South Africa

Full list of author information is available at the end of the article
}

application due to its simplicity, speed and relatively low cost, in comparison to the gold standard of phenotypic assays [4-6]. Furthermore, the prediction algorithms are continuously evaluated $[7,8]$, while mutation lists keep being updated to improve predictability of drug resistance $[9,10]$. Disparities between prediction methods have decreased but discordances still exist between the different algorithms, especially for some ARVs, as at 2015 [11]; which motivates the need to further improve accuracy.

Prediction accuracy is essential, as low-accuracy predictions increase the risk of prescribing sub-optimal drug regimens and missing the timing for regimen switches, leading to patients developing resistance 
sooner and so needing recourse to less well-tolerated third line ARV therapy. If left uncontrolled, the accumulation of resistance mutations may increase the probability of resistant strains directly spreading to drug-naive individuals, rendering therapy more difficult. In order to address these issues, different research groups have been involved in producing independent prediction algorithms - such as REGA [12], ANRS [13] and HIVdb [14] amongst others [15]. As stated in [17], to date the most widely used ones are the HIVdb algorithm [14] and the support vector machine-based geno2pheno tool $[16,18]$. More recent work has applied different machine learning approaches for drug resistance prediction, for instance multi-label classification [17], K-Nearest Neighbor and Random Forests [19], sparse signal representations coupled to Delaunay triangulation [20, 21] and Support Vector Machines variants [22], some of which are based on sequence information, while others also utilise protein structural information.

The objective of this work was to develop prediction models that are as accurate as possible. This problem is usually treated as one of classification, since in a clinical context it is normally sufficient to predict the effectiveness (or not) of a given ARV. However, here we solve a regression problem, thereby making full use of all available data and so potentially improving the predictive accuracy of the model. We note that the model output may be transformed into a classification by setting cutoff values, and that the drug resistance score may be clinically useful if the value is borderline, i.e. very close to a cut-off value.

Our method incorporated the following features: (a) The prediction algorithm used was a regression Artificial Neural Network (ANN); (b) because the great majority of publicly available data in the Stanford HIVdb is for subtype B HIV, only subtype B data was used in this database to train and test the network, so that the prediction algorithm is mainly applicable to subtype B sequence data; (c) in order to reduce data noise, various forms of data filtering, as described in the Methodology section, were used. Our regression ANN models compared favourably against recent work by Shen and coworkers [19], for which similar metrics were used. The ANN regression models were applied to the protease (PR) inhibitors fosamprenavir (FPV), atazanavir (ATV), indinavir (IDV), lopinavir (LPV), saquinavir (SQV), tipranavir (TPV), nelfinavir (NFV) and darunavir (DRV), and to the reverse transcriptase (RT) inhibitors lamivudine (3TC), abacavir (ABC), zidovudine (AZT), stavudine (D4T), didanosine (DDI), tenofovir (TDF), efavirenz $(\mathrm{EFV})$, etravirine (ETR), nevirapine (NVP), rilpivirine (RPV). Applying cut-offs, we obtain a classification output from our ANN models which is then evaluated against HIVdb and SHIVA [17]. Our work resulted in the production of drug-specific regression ANNs with high mean $R^{2}$ values, low variance and competitive classification performances for each of the eight PR inhibitors (PIs), six nucleoside RT inhibitors (NRTIs) and four non-nucleoside RT inhibitors (NNRTIs) for predictions from subtype B HIV.

\section{Methods}

\section{Dataset description}

Unfiltered PhenoSense assay datasets were retrieved from Stanford HIVdb [23] for both PR and RT. The datasets are compactly organized from a consensus B sequence with conserved positions coded as "-", with differing residues coded as the actual amino acids. Mixed residues are grouped together while indels are represented as "\#" and " $\sim$ " respectively in a tab-separated file format. Drug resistance scores for PR and RT inhibitors are present for each sequence entry as metadata.

\section{Dataset pre-processing}

Incomplete sequence entries (i.e. with missing fold resistance ratios for some ARVs) were retained to increase the sample size. Sequences containing the ambiguous residue ' $\mathrm{X}$ ', indels or the characters '.,' ',' 'l,' 'd' and '^' were flagged and then expanded to obtain all possible sequences consistent with the sequence data. The sequence expansion procedure thus yielded differing numbers of sequences for each ARV (Table 1). Non-B subtypes were also filtered out from the dataset to improve predictability for the subtype B cluster only. RT sequences were truncated to 240 residues to conform to the format of the filtered RT PhenoSense dataset as available from Stanford HIVdb. Several sequence entries yielded several thousand to millions of combinations of sequences, which made the initial design non-practical in terms of running time and also potentially introduced bias to the model that would be obtained from the dataset. This inherent uncertainty resides in the fact that the sequences may truly be mixed or contain sequencing errors. Thus a filter was introduced that removed from the datasets any sequence whose expansion yielded more sequences than some user-chosen cut-off value.

The experiment was initially started by training machine learners with sequences that had less than 5,10 , 20, 50, 100, 200, 300 and 1000 combinations upon expansion. Thereafter only the 300 and 1000 filter levels were used as candidates for rare variant filtering, due to their higher performance and number of unique sequence IDs that they contained. Rare variant filtering here means that a sequence is removed if it contains a residue at a given position that occurs only once across all sequence samples, and ANNs were constructed and tested both with and without this filtering. In order to process the sequence data, the amino acid letters were 
Table 1 ANN topologies and filtering parameters for highest observed accuracies for the various ARVs

\begin{tabular}{|c|c|c|c|c|c|c|}
\hline$\overline{\text { ARVs }}$ & & Topology & $\begin{array}{l}\text { Number of unique sequence } \\
\text { IDs/expanded sequences }\end{array}$ & Number of allowed combinations & Rare variant filtering & Number of outliers removed \\
\hline \multirow[t]{8}{*}{ Pls } & ATV & $10 \times 8 \times 6$ & $995 / 13,625$ & $<1000$ & $\checkmark$ & 1 \\
\hline & DRV & $8 \times 8$ & $590 / 10,374$ & $<1000$ & $\checkmark$ & 2 \\
\hline & FPV & $8 \times 8 \times 8$ & $1429 / 17,501$ & $<1000$ & $x$ & none \\
\hline & IDV & $8 \times 6 \times 10$ & 1459 / 16,977 & $<1000$ & $\checkmark$ & 1 \\
\hline & LPV & $10 \times 8 \times 10$ & $1284 / 11,019$ & $<300$ & $x$ & none \\
\hline & NFV & $10 \times 10 \times 10$ & $1524 / 11,929$ & $<300$ & $x$ & none \\
\hline & SQV & $10 \times 10 \times 8$ & $1484 / 11,509$ & $<300$ & $x$ & none \\
\hline & TPV & $10 \times 6 \times 8$ & $698 / 11,989$ & $<1000$ & $\checkmark$ & 2 \\
\hline \multirow[t]{6}{*}{ NRTIS } & $3 \mathrm{TC}$ & $10 \times 10 \times 6$ & $1342 / 33,181$ & $<1000$ & $\checkmark$ & none \\
\hline & $A B C$ & 14 & $1401 / 34,016$ & $<1000$ & $x$ & none \\
\hline & $\mathrm{AZT}$ & 19 & $1358 / 33,818$ & $<1000$ & $\checkmark$ & none \\
\hline & D4T & $10 \times 4 \times 4$ & $1365 / 34,056$ & $<1000$ & $\checkmark$ & none \\
\hline & DDI & $10 \times 6 \times 6$ & $1368 / 34,062$ & $<1000$ & $\checkmark$ & none \\
\hline & TDF & $10 \times 2$ & 1130 / 29,637 & $<1000$ & $x$ & none \\
\hline \multirow[t]{4}{*}{ NNRTIS } & EFV & $10 \times 6 \times 10$ & $1400 / 33,906$ & $<1000$ & $\checkmark$ & none \\
\hline & ETR & $8 \times 2 \times 10$ & $448 / 11,397$ & $<1000$ & $x$ & 2 \\
\hline & NVP & $10 \times 10 \times 4$ & 1414 / 20,348 & $<300$ & $x$ & none \\
\hline & RPV & 16 & 169 / 2977 & $<1000$ & $\checkmark$ & none \\
\hline
\end{tabular}

converted to integers using an ad hoc Python script, utilizing a simple integer encoding scheme, whereby resi-

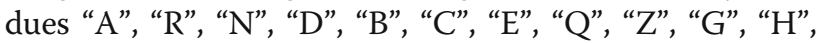
"I", "L", "K", "M", "F", "P", "S", “T", "W", "Y" and "V" were converted to positive integers 1 to 22 respectively in a similar manner, but not identical to the encoding approach used by Araya and Hazelhurst [4], who applied codon-based integer encoding instead on a dataset used by Ravela and coworkers in 2003 [24]. Possible outliers were detected by using (1) Principal Components Analysis from input features and target values and (2) the prediction error distributions between actual and predicted scores, and removed (Table 1).

\section{Neural network construction and architecture optimization}

MATLAB's (version 2016a) implementation of the Levenberg-Marquardt feed-forward algorithm with backpropagation from the Neural Network Toolbox was used for supervised training, utilizing the mean squared error (MSE) for weight adjustment. Absolutely conserved residue positions were filtered out in order to reduce computation time. The initial dataset was (pseudo) randomly split into training, testing and validation sets at rates of $70 \%, 15$ and 15\% respectively, setting random seed numbers for reproducibility in training and cross-validation. Training was stopped upon reaching any of a maximum of 1000 epochs, a maximum of 6 successive validation failures to decrease or a performance gradient lower than a minimum set at $1 \mathrm{e}-7$. Input features were the 1letter amino acid characters recoded as integers while the target values were the individual fold drug resistance ratios. After initial runs using all drug target values at once for training the regression model, large MSE values were obtained (not shown), which redirected analysis towards building individual trained matrices for each drug target. As a requirement for the MATLAB's newff function, both the feature vectors and their matching target values were transposed. The number of hidden layers was varied from 1 to 3 while nodes were set at permutations of 2, 4, 6, 8 and 10 for each hidden layer. One hidden layer of 5-20 nodes was re-evaluated in cases where high training performances were observed to have a significantly lower test performances or high variances.

\section{Evaluation of training performance}

Training performance was assessed both by regression and classification methods. For regression-based evaluation, the coefficient of determination $\left(R^{2}\right)$ values were obtained between the predicted $\left(y_{i}\right)$ and actual $\left(x_{i}\right)$ fold scores for the whole dataset using the formula

$$
R^{2}=\frac{\left[n\left(\sum_{i=1}^{n} x_{i} y_{i}\right)-\left(\sum_{i=1}^{n} x_{i}\right)\left(\sum_{i=1}^{n} y_{i}\right)\right]^{2}}{\left[n \sum_{i=1}^{n} x_{i}^{2}-\left(\sum_{i=1}^{n} x_{i}\right)^{2}\right]\left[n \sum_{i=1}^{n} y_{i}^{2}-\left(\sum_{i=1}^{n} y_{i}\right)^{2}\right]}
$$

Further, the dataset was randomly divided into 5 subsets of approximately equal size, and 5 different ANNs 
were trained on datasets that comprised 4 of the 5 subsets, and then 5 different $R^{2}$ values were calculated; we then calculated the mean and the standard deviation of these $5 R^{2}$ values. Regression performances were then compared against prediction models from the article published in 2016 by Shen and co-workers [19], in which regression machine learning models, namely the Random Forest and the K-nearest neighbor algorithms were used. The raw dataset used in this work and in ref. [19] is the same, i.e. the Stanford HIVdb dataset; however, the filtering used in this paper is as described above, whereas ref. [19] uses filtering provided by Stanford HIVdb [23]. In order to further verify our models against overfitting, $\mathrm{R}^{2}$ values were calculated over different subsets of the data set, namely the whole dataset, the validation set and finally the test set.

Furthermore, classification accuracy was evaluated against Stanford HIVdb and a recently-published approach implemented as the SHIVA web server [17]. We used the EMBOSS backtranseq tool [25] to back-translate protein sequences to one of its (DNA) codon permutations in FASTA format as input for Stanford HIVdb's Sierra web service (GraphQL API) tool to obtain resistance predictions. SHIVA predictions were obtained by submitting FASTA-formatted protein sequences to the web server. Drug resistance classes (susceptible, resistant and intermediate) were coded as numbers 0,1 and 2 respectively. While Stanford HIVdb defined three classes, SHIVA defined two: susceptible and resistant. Classification accuracies were evaluated by calculating misclassification rates, defined as the proportion of non-concordant pairs between PhenoSense Assay classes and the independentlypredicted classes for each of: our ANN approach, Stanford HIVdb and SHIVA. Cut-offs from Stanford HIVdb available at [26] were used for classifying our ANN predictions and those of the PhenoSense Assay dataset. We do not define new binary cut-offs for evaluating SHIVA; for a limited number of ARVs binary cut-offs are available from the PhenoSense Assay [27], and for the remaining ARVs we proceed in the following way. An upper and a lower bound misclassification rate were computed for SHIVA as the conversion from a multiclass to a binary classification is ambiguous - an intermediate class may lie closer to a resistant or susceptible class. We set the number of truly misclassified pairs $(0,1$ or 1,0$)$ as the lower bound, while the number of discordant pairs involving intermediate resistance sequences $(2,0$ or 2,1$)$ was added to the discordance value to set an upper bound for misclassification rates. All proportions were then evaluated as percentages, as shown in Table 2.

\section{Results and discussion}

Table 1 shows that differing numbers of sequences were obtained from the different filtering approaches. In general, allowing expansion of sequences to less than 1000,
Table 2 Comparison of misclassification rates (percentages) for our ANN approach, Stanford HIVdb and SHIVA

\begin{tabular}{lllll}
\hline ARVs & & ANN & HIVdb & SHIVA \\
\hline PIs & ATV & 26.61 & 28.57 & 84.53 \\
& DRV & 2.98 & 22.57 & $32.41-53.49$ \\
& FPV & 16.08 & 36.97 & $67.0-79.74$ \\
& IDV & 34.29 & 26.19 & 81.92 \\
& LPV & 9.79 & 36.82 & $68.05-83.51$ \\
& NFV & 25.23 & 20.36 & 80.84 \\
NRTIs & SQV & 30.37 & 38.75 & $67.25-88.16$ \\
& TPV & 9.07 & 39.88 & unavailable \\
& 3TC & 3.87 & 12.09 & 90.21 \\
& ABC & 6.53 & 33.78 & $50.76-72.25$ \\
& AZT & 36.19 & 29.88 & 90.38 \\
& D4T & 7.31 & 44.07 & 79.15 \\
& DDI & 8.05 & 57.52 & $34.14-92.44$ \\
NNRTIS & TDF & 5.39 & 37.2 & $37.36-66.53$ \\
& EFV & 16.08 & 21.05 & 81.32 \\
& ETR & 6.58 & 13.21 & unavailable \\
& NVP & 24.87 & 9.4 & 73.97 \\
& RPV & 1.55 & 24.99 & 8.33 \\
\hline
\end{tabular}

combined with rare variant filtering produced the best results. Multiple (2-3) hidden layers were found to be required for all ARVs, with the exception of ABC, AZT, and RPV. DRV, ETR and RPV have the lowest numbers of unique sequence IDs, and hence may suffer from lack of generalizability compared to the other ARVs. However, in this study we attempted to find the optimal balance between the number of sequences and the possibility of retaining sequences containing sequencing errors.

The procedure used to build our models is referred to as protocol A. Our results are compared to the models used by Shen and co-workers [19], namely the Random Forest (RF) and the K-nearest neighbor (KNN), which both utilise Delaunay triangulation for structural feature encoding (henceforth referred to as protocol $\mathrm{B}$ and $\mathrm{C}$ respectively in this paper).

\section{Regression performances for HIV Pls}

The results are presented in Fig. 1a and Additional file 1: Table S1. The procedure used to build our models is referred to as protocol A. In all, protocol A yielded better results than protocols $B$ and $C$. Very low variances were generally observed using protocol $\mathrm{A}$, except in the case of ATV, IDV and LPV where variances were comparable to those observed in protocols B and C. Improvements of largest magnitudes for PIs were observed from protocol A for FPV, SQV and TPV with mean differences of 0.117 , 0.116 and 0.219 respectively from the top-scoring protocols in $\mathrm{B}$. 


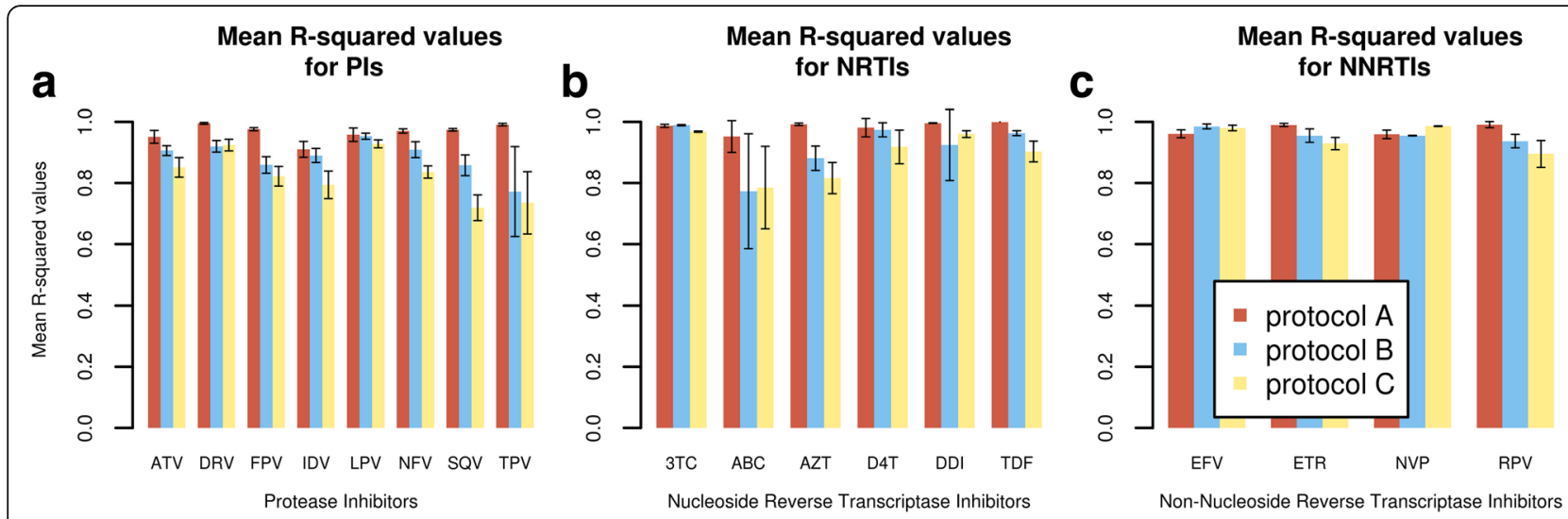

Fig. 1 The mean $R^{2}$ values and their standard deviations for the protocols $A, B, C$, and the various ARVs

\section{Regression performances for NRTIs}

In the case of NRTIs (Fig. $1 \mathrm{~b}$ and Additional file 1: Table S2), better predictability was observed for all drugs using protocol A except for 3TC, where the performance, though high, was similar to that obtained in protocol B. Very high mean $\mathrm{R}^{2}$ values with very small variances were obtained for AZT, DDI and TDF. Their high degree of fit combined to their low variability suggests that the ANN model is explaining most of the observed variation, likely due to higher sequence quality obtained after filtering.

\section{Regression performances for NNRTIs}

In the case of NNRTIs (Fig. 1c and Additional file 1: Table S3), protocol C outperformed protocol A by a narrow margin in for EFV and NVP. Very high mean accuracies were attained in the case of RPV and ETR, surpassing both protocols B and C. However, the smaller sample size for RPV (Table 1) (169 unique sequence IDs for a total of 2977 expanded sequences) may indicate that while appearing to perform exceptionally well, the model may not generalize well to more divergent sequences. ETR is supported by a comparatively higher number of unique sequence IDs, and will generalize slightly better that the model developed for RPV.

\section{Overfitting assessment}

As seen in Table 3, for all ARVs we verify that overfitting is minimized by ensuring that $\mathrm{R}^{2}$ values do not significantly decline in the test set with respect to both the whole dataset and the validation sets.

\section{Classification performance for all antiretrovirals}

We provide additional support for our approach by comparing misclassification rates against Stanford HIVdb and SHIVA, all with respect to the PhenoSense assay data. It can be observed from Table 2 that lower misclassification rates are obtained, with the exception of NVP, AZT, NFV and IDV. An important point to observe here is that we considered the entirety of the dataset filtered by our means for the development of the ANN described in this paper, the counts being shown in Table 1. This was performed so that only high confidence sequences would be compared for each individual antiretroviral. Both Stanford HIVdb and SHIVA were developed using another data set, the Stanford HIVdb pre-filtered data, and this factor may have affected their performance on the dataset used here.

Table $3 R^{2}$ values (3 dp) obtained from individual subsets obtained after filtering

\begin{tabular}{lllll}
\hline ARV classes & ARVs & Whole dataset $R^{2}$ values & $\begin{array}{l}\text { Validation set } \\
R^{2} \text { values }\end{array}$ & $\begin{array}{l}\text { Test set } \\
R^{2} \text { values }\end{array}$ \\
\hline PIs & ATV & 0.951 & 0.913 & 0.856 \\
& DRV & 0.991 & 0.991 & 0.989 \\
& FPV & 0.980 & 0.938 & 0.958 \\
& IDV & 0.899 & 0.816 & 0.842 \\
& LPV & 0.966 & 0.922 & 0.883 \\
& NFV & 0.975 & 0.924 & 0.939 \\
& SQV & 0.977 & 0.949 & 0.906 \\
& TPV & 0.989 & 0.995 & 0.943 \\
& 3TC & 0.995 & 0.988 & 0.985 \\
& ABC & 0.984 & 0.956 & 0.954 \\
& AZT & 0.994 & 0.979 & 0.985 \\
& D4T & 0.995 & 0.996 & 0.979 \\
& DDI & 0.997 & 0.997 & 0.992 \\
& TDF & 0.999 & 1.000 & 0.992 \\
& EFV & 0.976 & 0.905 & 0.967 \\
& ETR & 0.996 & 0.993 & 0.982 \\
& NVP & 0.962 & 0.939 & 0.927 \\
& RPV & 0.982 & 0.956 & 0.915 \\
\hline
\end{tabular}




\section{Conclusions}

This work focused on the pre-processing and optimization of ANN regression models for the prediction of fold resistance scores for $\mathrm{HIV}-1$ subtype $\mathrm{B}$ using $\mathrm{RT}$ and PR PhenoSense data available in the public domain from Stanford HIVdb. As expressed by Dahake and co-workers [28], there is a need to develop subtype-specific databases, and we made such an attempt by constraining the dataset for subtype specificity, sacrificing generalizability for a higher predictive performance for subtype B. The results obtained show that the predictive quality of the ANN regression models is at least comparable to that of other methods, and for most ARVs is a definite improvement.

The approach presented in this paper is applicable to subtype $B$, and an obvious question is whether it can be extended to the other subtypes? Previous studies $[29,30]$ involving HIV-1 subtypes A, B and C envelope glycoprotein V3 loop region, suggest that subtype $B$ and $C$ share similar co-receptor usage as opposed to subtype A. Also, Raymond and co-workers [31] hinted that subtypes B and $\mathrm{C}$ share similar genotypic determinants, and for this reason, by extrapolation our method may extend to the C subtype. However, a key difficulty is the paucity of publicly available phenotypic assay data for training and testing any extrapolation to other subtypes, so the development of a methodology that leads to accurate models will be challenging [32, 33]. It is hoped that our work will lead to more non-B subtype drug resistance data becoming available.

\section{Additional file}

Additional file 1: Table S1. Mean R2 values and their standard deviations for Pls for protocols A, B and C. Table S2. Mean R2 values and their standard deviations for NRTIs for protocols A, B and C. Table S3. Mean R2 values and their standard deviations for NNRTls for protocols A, B and C. (DOC $45 \mathrm{~kb})$

\section{Abbreviations}

3TC: Lamivudine; ABC: Abacavir; ANN: Artificial neural network; ANRS: Agence Nationale de Recherche sur le Sida et les hepatites virales; ARV: Antiretroviral; ATV: Atazanavir; AZT: Zidovudine; D4T: Stavudine; DDI: Didanosine;

DRV: Darunavir; EFV: Efavirenz; ETR: Etravirin; FPV: Fosamprenavir; HIV: Human immunodeficiency virus; HIVdb: HIV drug resistance database; IDV: Indinavir; KNN: K-Nearest Neighbors; LPV: Lopinavir; MSE: Mean squared error; NFV: Nelfinavir; NNRTI: Non-nucleoside reverse transcriptase inhibitor; NRTI: Nucleoside reverse transcriptase inhibitor; NVP: Nevirapine; Pl: Protease inhibitor; RF: Random forest; RPV: Rilpivirine; RT: Reverse transcriptase; SQV: Saquinavir; TDF: Tenofovir; TPV: Tipranavir
\end{abstract}

\section{Acknowledgements}

Not applicable.

\section{Funding}

This work was supported by the National Research Foundation of South Africa under grant number 93690 awarded to ÖTB, and by grant number 80983 awarded to NTB. The content is solely the responsibility of the authors and does not necessarily represent the official views of the funders.

\section{Availability of data and materials}

The datasets analysed during the current study are available in the Stanford HIVdb repository, https://hivdb.stanford.edu/pages/genopheno.dataset.html [23].

\section{Authors' contributions}

OSA wrote the scripts for filtering and computing the neural networks, and drafted the manuscript. ÖTB and NTB helped in the design of the study, in analysing the results, and in revising the manuscript drafts. All authors read and approved the final manuscript.

\section{Authors' information}

O.S.A. completed his undergraduate studies with Honours in Agricultural Biotechnology at the University of Mauritius. He later joined the Research Unit in Bioinformatics (RUBi) while doing his Master's degree at Rhodes University in South Africa, where he is currently doing his PhD. His research is focused around the application of residue interaction networks and the use artificial neural networks in the context of drug resistance prediction in HIV

N.T.B. studied Mathematics, receiving his BA and MA degrees from the University of Cambridge, U.K., and PhD from the University of Southampton, U.K. He has held positions of Professor of Applied Mathematics for many years.

Ö.T.B. received her BSC degree in Physics from Bogazici University, Istanbul, Turkey. Then she moved to the Department of Molecular Biology and Genetics at the same University for her MSc degree. She obtained her PhD from Max-Planck Institute for Molecular Genetics and Free University, Berlin, Germany. She is the Director of Research Unit in Bioinformatics (RUBi) at Rhodes University. Özlem's broad research interest is comparative genomics, structural bioinformatics and tool development.

\section{Ethics approval and consent to participate}

Not applicable.

\section{Consent for publication}

Not applicable.

\section{Competing interests}

The authors declare that they have no competing interests.

\section{Publisher's Note}

Springer Nature remains neutral with regard to jurisdictional claims in published maps and institutional affiliations.

\section{Author details}

${ }^{1}$ Research Unit in Bioinformatics (RUBi), Department of Biochemistry and Microbiology, Rhodes University, Grahamstown 6140, South Africa. ${ }^{2}$ Department of Mathematics (Pure and Applied), Rhodes University, Grahamstown 6140, South Africa.

Received: 11 March 2017 Accepted: 7 August 2017

Published online: 15 August 2017

\section{References}

1. Reynolds L. HIV as a chronic disease considerations for service planning in resource-poor settings. Glob Health. 2011;7:35.

2. Zhang F, Dou Z, Ma Y, Zhang Y, Zhao Y, Zhao D, et al. Effect of earlier initiation of antiretroviral treatment and increased treatment coverage on HIV-related mortality in China: a national observational cohort study. Lancet Infect Dis. 2011:11:516-24.

3. Xing H, Ruan $Y$, Li J, Shang H, Zhong P, Wang $X$, et al. HIV drug resistance and its impact on antiretroviral therapy in Chinese HIV-infected patients. PLoS One. 2013;8:1-7.

4. Araya ST, Hazelhurst S. Support vector machine prediction of HIV-1 drug resistance using the viral nucleotide patterns. Trans R Soc South Africa. 2009;64:62-72

5. Tang MW, Shafer RW. HIV-1 antiretroviral resistance: Scientific principles and clinical applications. Drugs. 2012;72:1-25.

6. Prosperi MCF, De Luca A. Computational models for prediction of response to antiretroviral therapies. AIDS Rev. 2012;14:145-53.

7. Drăghici S, Potter RB. Predicting HIV drug resistance with neural networks. Bioinformatics. 2003;19:98-107. 
8. Riemenschneider M, Heider D. Current Approaches in Computational Drug Resistance Prediction in HIV. Curr HIV Res 2016;1-9.

9. Wensing AM, Calvez V, Günthard HF, Johnson VA, Paredes R, Pillay D, et al. 2014 update of the drug resistance mutations in HIV-1. Top. Antivir. Med. 2014;22:642-50

10. Wensing AM, Calvez V, Günthard HF, Johnson VA, Paredes R, Pillay D, et al. 2015 update of the drug resistance mutations in HIV-1. Top Antivir Med. 2015;23:132-41.

11. Wagner S, Kurz M, Klimkait T. Algorithm evolution for drug resistance prediction: comparison of systems for HIV-1 genotyping. Antivir Ther. 2015;20:661-5.

12. Van Laethem K, De Luca A, Antinori A, Cingolani A, Perno CF, Vandamme AM. A genotypic drug resistance interpretation algorithm that significantly predicts therapy response in HIV-1-infected patients. Antivir Ther. 2002;7:123-9.

13. Meynard J-L, Vray M, Morand-Joubert L, Race E, Descamps D, Peytavin G, et al. Phenotypic or genotypic resistance testing for choosing antiretroviral therapy after treatment failure: a randomized trial. AIDS. 2002;16:727-36.

14. Rhee S-Y, Gonzales MJ, Kantor R, Betts BJ, Ravela J, Shafer RW. Human immunodeficiency virus reverse transcriptase and protease sequence database. Nucleic Acids Res. 2003:31:298-303.

15. Lengauer T, Sing T. Bioinformatics-assisted anti-HIV therapy. Nat Rev Microbiol. 2006:4:790-7.

16. Liu TF, Shafer RW. Web resources for HIV type 1 genotypic-resistance test interpretation. Clin Infect Dis. 2006:42:1608-18.

17. Riemenschneider M, Hummel T, Heider D. SHIVA - a web application for drug resistance and tropism testing in HIV. BMC Bioinf. 2016;17:314.

18. Beerenwinkel N, Schmidt B, Walter H, Kaiser R, Lengauer T, Hoffmann D, et al. Geno2pheno: interpreting genotypic HIV drug resistance tests. IEEE Intell Syst Their Appl. 2001;16:35-41.

19. Shen C, Yu X, Harrison RW, Weber IT. Automated prediction of HIV drug resistance from genotype data. BMC Bioinf. 2016;17:278.

20. Yu X, Weber IT, Harrison RW. Sparse representation for prediction of HIV-1 protease drug resistance. Proc. 2013 SIAM Int. conf. Data mining. SIAM Int. conf. Data Min. 2013;2013:342-9.

21. Yu X, Weber IT, Harrison RW. Prediction of HIV drug resistance from genotype with encoded three-dimensional protein structure. BMC Genomics. 2014;15:S1.

22. Masso M, Vaisman II. Sequence and structure based models of HIV-1 protease and reverse transcriptase drug resistance. BMC Genomics. 2013;14(Suppl 4):S3.

23. Stanford HIVdb. Genotype-Phenotype Datasets. 2014 [cited 2016 Dec 13]. Available from: https://hivdb.stanford.edu/pages/genopheno.dataset.html.

24. Ravela J, Betts BJ, Brun-Vézinet F, Vandamme A-M, Descamps D, van Laethem K, et al. HIV-1 protease and reverse transcriptase mutation patterns responsible for discordances between genotypic drug resistance interpretation algorithms. J Acquir Immune Defic Syndr. 2003;33:8-14.

25. Rice P, Longden I, Bleasby A. EMBOSS: the European molecular biology open software suite. Trends Genet. 2000;16:276-7.

26. Hedlin H. Genotype-Phenotype Datasets: DRMcv. 2014 [cited 2017 May 22]. Available from: https://hivdb.stanford.edu/download/GenoPhenoDatasets/ DRMCV.R.

27. Monogram Biosciences. Phenosense HIV Drug Resistance Assay. 2014 [cited 2017 Jul 18]. p. 1-2. Available from: https://www.monogrambio.com/sites/ monogrambio/files/imce/uploads/PS_report_new_Watermark.pdf.

28. Dahake R, Mehta S, Yadav S. Polymorphisms in HIV-1 subtype $\mathrm{C}$ reverse transcriptase and protease genes in a patient cohort from Mumbai. J Antivir Antiretrovir. 2016;8:5-7.

29. Gupta S, Neogi U, Srinivasa H, Shet A. Performance of genotypic tools for prediction of tropism in HIV-1 subtype C V3 loop sequences. Intenvirology. 2015;58:1-5.

30. Riemenschneider M, Cashin KY, Budeus B, Sierra S, Shirvani-Dastgerdi E, Bayanolhagh S, et al. Genotypic prediction of co-receptor tropism of HIV-1 subtypes a and C. Sci Rep. 2016;6:24883.

31. Raymond S, Delobel P, Mavigner M, Ferradini L, Cazabat M, Souyris C, et al Prediction of HIV type 1 subtype $C$ tropism by genotypic algorithms built from subtype B viruses. J Acquir Immune Defic Syndr. 2010;53:167-75.

32. Awoke T, Worku A, Kebede Y, Kasim A, Birlie B, Braekers R, et al. Modeling Outcomes of First-Line Antiretroviral Therapy and Rate of CD4 Counts Change among a Cohort of HIV / AIDS Patients in Ethiopia: A Retrospective Cohort Study. PLoS ONE. 2016;11:1-18.

33. Duber HC, Dansereau E, Masters SH, Achan J, Burstein R, DeCenso B, et al. Uptake of WHO recommendations for first-line antiretroviral therapy in Kenya, Uganda, and Zambia. PLoS One. 2015;10:1-12.

\section{Submit your next manuscript to BioMed Central and we will help you at every step:}

- We accept pre-submission inquiries

- Our selector tool helps you to find the most relevant journal

- We provide round the clock customer support

- Convenient online submission

- Thorough peer review

- Inclusion in PubMed and all major indexing services

- Maximum visibility for your research

Submit your manuscript at www.biomedcentral.com/submit
Biomed Central 